\title{
Ego-depletion or invigoration in solving the tower of Hanoi? Action orientation helps overcome planning deficits
}

\author{
Miguel Kazén ${ }^{1}$ [D $\cdot$ Julius Kuhl ${ }^{1}$ \\ Published online: 1 May 2020 \\ (C) The Author(s) 2020, corrected publication 2021
}

\begin{abstract}
Personality systems interaction (PSI) theory postulates two executive control modes: Self-control and self-regulation. Selfcontrol, typical of state oriented persons, should result in "ego depletion" whereas self-regulation, typical of action oriented persons, should result in invigoration. State- and action-oriented participants performed the Plan-a-Day and the 5-disk Tower-ofHanoi tasks. There were no differences between them on the first task, but action had better performance than state oriented in terms of number of moves and solution time on the second task, independently of differences in self-determination. Better performance in the Tower of Hanoi correlated positively with activation, and negatively with apathy. We conclude that whereas self-control is associated with depletion, self-regulation is associated with invigoration in performing the Tower of Hanoi task.
\end{abstract}

Keywords PSI theory $\cdot$ Ego-depletion $\cdot$ Action orientation $\cdot$ Self-control $\cdot$ Self-regulation $\cdot$ Tower of Hanoi $\cdot$ Self-determination

Exertion of self-control is proposed to deplete resources needed to carry out subsequent effortful tasks. This has been called "ego depletion" and it manifests itself in loss of energy and lowered performance on subsequent tasks requiring self-control. Everyday examples of these tasks are overcoming procrastination in doing intended tasks, restraint in alcohol or food consumption, or stop verbal insults or violent acts to others. Those behaviors are less likely to occur after the person has exerted self-control for a while (Baumeister et al. 2000; Tice and Bratslavsky 2000), suggesting ego-depletion. Baumeister et al. (1998) proposed a limited-resource strength model as explanation of this effect. It asserts that an initial exertion of self-control drains the limited energy or strength that one has and needs to carry out additional activities, with a subsequent drop in performance or deficits in behaviors requiring impulse control. Exertion of self-control can occur not only as a result of restrain in carrying out a preferred behavior but also by making choices among different alternatives (Baumeister et al. 1998). Moreover, ego-depletion not only

Miguel Kazén

mikazen@uos.de; mikazen@gmx.de

Julius Kuhl

jkuhl@uos.de

1 Faculty of Human Sciences, Institute of Psychology, University of Osnabrück, 49074 Osnabrück, Germany reduces performance in tasks requiring impulse control but also induces "mental passivity", a preference for passive behaviors, such as resting and watching TV (Vonasch et al. 2017).

Although there is evidence for volitional depletion in the literature (see Hagger et al. 2010, for a meta-anaylsis), more research is needed to investigate what it takes to overcome or prevent volitional deficits related to previous exertion of selfcontrol. Subsequent to the original studies, several authors proposed moderators of ego-depletion. One of them is autonomy, which is a central concept in self-determination theory (SDT; Ryan and Deci, 2000, 2008). According to SDT, ego-depletion effects should occur only when our behavior is externally controlled, but not when it is autonomous and self-integrated. Muraven (2008) found that autonomously motivated self-control is less depleting than self-control done for extrinsic reasons (see also Muraven et al. 2008). Moller et al. (2006) working within the perspective of SDT distinguished between controlled vs. autonomous choices. In three experiments Moller et al. found evidence of ego depletion when participants' choices were externally controlled but not when they were carried out in an autonomous, self-determined form. Participants showed higher persistence in subsequent tasks under conditions of autonomy.

Ego-depletion effects are expected to occur in all persons, however, there is increasing evidence suggesting that they are moderated by personality. Specifically, action vs. state orientation, a personality disposition related to volitional 
control, has been proposed as moderator of ego-depletion effects (Gröpel et al. 2014; Kazén et al. 2015). Personality systems interactions (PSI) theory distinguishes two executive modes related to volitional enactment of difficult tasks: Selfcontrol and self-regulation (Kuhl 2000, 2001; Kuhl and Fuhrmann 1998). Self-control is an externally-controlled mode, whereas self-regulation is a more democratic, autonomous, mode. Presumably, the individual suppresses her/his own needs to reach an assigned goal in the self-control mode only. According to this hypothesis, the self-control mode is associated with effort and depletion because behavioral energy has to work against negative incentives rather than being supported by positive incentives. Self-regulation, in contrast, is a more self-congruent mode to carry out our intentions, and it is not associated with effortful enactment (see also Baumeister et al. 2007).

Fuhrmann and Kuhl (1998) investigated enactment of healthy-eating intentions during two weeks in two groups of participants: Those with a personality disposition towards self-control or self-regulation. Executive mode (self-control or self-regulation) and instructional feedback interacted. For self-controlled participants higher goal enactment was found with self-punishment (punishing oneself for not keeping up the intended eating behaviors) whereas for self-regulation participants higher goal enactment was found with self-reward (rewarding oneself for keeping up the intended eating behaviors). That is, self-punishment (effortful) was more effective for goal enactment for the self-controlled group, whereas selfreward (less effortful) was more effective for the selfregulation group.

There is a great amount of evidence on the association between self-regulation and action orientation and between self-control and state orientation (Kuhl and Beckmann 1994; see also Goschke and Kuhl 1993; Heckhausen \& Strang, 1988; Jostmann and Koole 2007; Kazén et al. 2008; Koole and Jostmann 2004; Koole et al. 2012). People with high scores in the action orientation scale (Kuhl 1994) are classified as action oriented whereas those with low scores are classified as state oriented. Action oriented persons are postulated to have high levels of volitional efficiency, because they are able to self-generate positive affect when a task gets difficult or boring (Koole and Jostmann 2004). They work under the self-regulation mode. State oriented persons, on the other hand, are not able to self-generate positive affect to carry out the tasks. They work under the self-control mode (Beckmann and Kuhl 1984; Koole and Jostmann 2004; Kuhl 2000, 2001).

Heckhausen and Strang (1998) found that semiprofessional action-oriented basketball players had better performance (attempted shots, hits, and less dribbling errors) and exerted less physical effort (lower lactate concentrations and heart rates) than state-oriented players, under normal and record performance instructions. The authors suggested that actionoriented players have better "exertion control" in their sport performance than state-oriented players do. Kazén et al. (2008) found performance deficits in state-oriented persons under demanding conditions (i.e., those dampening positive affect or inducing a demanding intention to be executed later in the experiment) in (a) self-initiation of intentions in a prospective memory task, and in (b) self-choice decisions in a task in which they had to choose which of two goals to pursue, compared to an external-cue condition (where a cue suggested which goal to pursue). In contrast, action-oriented participants had significantly better performance in those tasks, especially under demanding conditions. More related to the present research, Jostmann and Gieselmann (2014) used a 3-disk computerized version of the Tower of Hanoi, with a series of trials, presenting first two nonconflict and then two conflict trials, and compared performance of action and state oriented persons under a control and a demanding condition. As predicted, Jostmann and Gieselmann found better performance (number of moves beyond the minimum and solution time) for action oriented compared to state oriented individuals under demanding conditions. On the other hand, "In a nondemanding control condition, state-oriented individuals performed equally well as action-oriented individuals." (op.cit., p. 399).

Note that in the experiments described in the last paragraph the authors presented only one task to assess performance and manipulated demands in different ways. Studies investigating performance deficits in state vs. action orientation using two tasks are seldom (for exceptions see Gröpel et al. 2014; Kazén et al. 2015). In a study dealing with ego depletion and action orientation carried out in China (Dang et al. 2015) the authors presented first a Stroop task and then a resource-demanding concentration task, to assess performance deficits. They found egodepletion - higher number of errors in the concentration task - in state oriented participants only.

In the present experiment we will test directly whether action oriented persons are more resistant to ego-depletion effects than state oriented, using two resource-demanding tasks related to planning behavior and executive processes: Plan-a-Day and a computerized Tower of Hanoi task. In contrast to Jostmann and Gieselmann (2014), (a) we used a typical ego-depletion paradigm (cf. Baumeister et al. 2000; Dang et al. 2015; Gröpel et al. 2014), exposing action and state oriented participants to two conceptually similar but resource demanding tasks, (b) to avoid possible carry-over effects of nonconflict and conflict trials, we applied here only one conflict trial, and (c) we increased difficulty by using the 5-disk, instead of the 3-disk version of the Tower of Hanoi. Other differences with Jostmann and Gieselmann (2014) are that (a) we investigated the role of autonomy in the performance of the Tower of Hanoi and (b) we examined the effect of mood (listlessness and activation) on this task, which is known to affect performance of state-oriented persons (cf. Kazén et al. 2008). 
Our first hypothesis is that only state oriented participants will show performance deficits (i.e., ego-depletion) in the second - Tower of Hanoi - task whereas action oriented participants will not show performance deficits in that task. We do not expect to find differences between them in the first - "Plana-Day" - task. Moreover, we expect this effect to occur independently of individual differences in autonomy. Our second hypothesis is that better performance in the Tower of Hanoi task will correlate positively with mood measures of activation (invigoration) and negatively with mood measures of apathy (depletion).

\section{Method}

\section{Participants}

In the experiment participated 34 undergraduate volunteers (21 women and 13 men), who received course credit for their participation. Mean age was 24.9 years (range: 19 to 42 years). Applying the norms (Kuhl 1994, p. 54) we classified 17 as action oriented on the decision (and initiative) dimension of the action control scale because their score was above the median (i.e., equal or higher to 6 , indicating stronger decisiveness; $M=8.2, S D=1.8$ ) and 17 as state oriented because their score was below the median (i.e., a score of 5 or lower; $M=$ 2.6, $S D=1.6$ ), indicating stronger hesitation.

\section{Questionnaires}

Action Control Scale This is a trait measure of volitional control (ACS; see Kuhl 1994). Example item: When I am getting ready to tackle a difficult problem: (a) I look for a way that the problem can be approached in a suitable manner, or (b) It feels like I am facing a big mountain that I don't think I can climb." In this example, option ( $a$ ) illustrates the action-oriented and (b) the state-oriented response alternative. Scores have a range of 0 to 12 , with higher values indicating action orientation (initiative or decisiveness) and lower values state orientation (inaction or indecisiveness). The scale has good reliability (Cronbach's alpha $=.78$; see Diefendorff et al. 2000, for psychometric information on the English version) and a wellestablished construct validity in research and applied settings (Kuhl and Beckmann 1994; see also Heckhausen \& Strang, 1988; Goschke and Kuhl 1993; Jostmann and Gieselmann 2014; Kazén et al. 2008; Kehr 2004; Koole and Jostmann 2004; Koole et al. 2012).

Mood Measures We administered a 21-item self-report mood inventory ("Right now, I feel ...."), using a 4-point Likert scale (from $1=$ not at all, to $4=$ completely) (similar to that of PANAS; cf. Watson et al. 1988). From this inventory three scales, each with three items, were relevant to our investigation: "Happiness" (as an index of positive affect; Cronbach's alpha $=.85$ ), "Activation" (as an index of invigoration; Cronbach's alpha $=.72$ ) and "Listlessness" (as an index of "apathy"; Cronbach's alpha = .78).

Self-Determination Scale We applied the Self-Determination scale of the Volitional Components Inventory (VCI; Kuhl and Fuhrmann 1998) with four items (Cronbach's alpha = .76). Example items are: "In doing what I do, I feel it was me who chose to do it." and "In most situations, I feel free to do what I think is right." The person responds to the degree of applicability of the statement to her/himself using a 4-point Likert scale: (from $1=$ not at all, to $4=$ completely). The self-determination scale correlates substantially with measures of Autonomy developed by Deci and Ryan (data obtained by Nicola Baumann with 80 participants). In particular, with the "General Causality Orientation-Autonomy" scale (see Deci and Ryan 1985): $r(78)=+.44, p<.0001$ and with the "Self-Determination Index" (see Ryan and Connell 1989): $r(78)=+.55, p<.0001$.

\section{Experimental Design}

We used a $2 \times 2$ mixed factorial design. The first, betweenparticipant factor, was Personality (state, action orientation) and the second, within-participant factor, was Planning Task (Plan-a-Day, Tower of Hanoi), which we manipulated. We had an equal number of state and action oriented participants in the study, and, because of the logic of volitional depletion investigations, we did not counterbalance the tasks, but kept the sequence of presentation constant: Plan-a-Day and Tower of Hanoi, respectively. We expected to find a significant interaction between Personality and Planning Task.

\section{Tasks}

Plan-a-Day Plan-a-Day is a computer-based scheduling task, related to a working situation (Funke and Krüger 1993, 1995), which can also be used for neuropsychological diagnosis of planning abilities (Holt et al. 2011). In the research version of this task participants are requested to imagine that they work for a company where they have to plan activities for a day, some of which have to be carried out at particular scheduled times. Information about the to-be-carried-out tasks for each day is listed on the right side of the screen. The different locations, the distances between locations, and the current position are shown in the left side (see Fig. 1 in Holt et al. 2011). Constraints for solving Plan-a-Day problems include earliest start, latest finish, location, duration of tasks, and distance between locations. Participants are asked to plan their daily activities before they implement their plan, which they do so by moving the symbol representing their current position and clicking it towards corresponding locations on the map. If they are too late in carrying out a task at a location, 


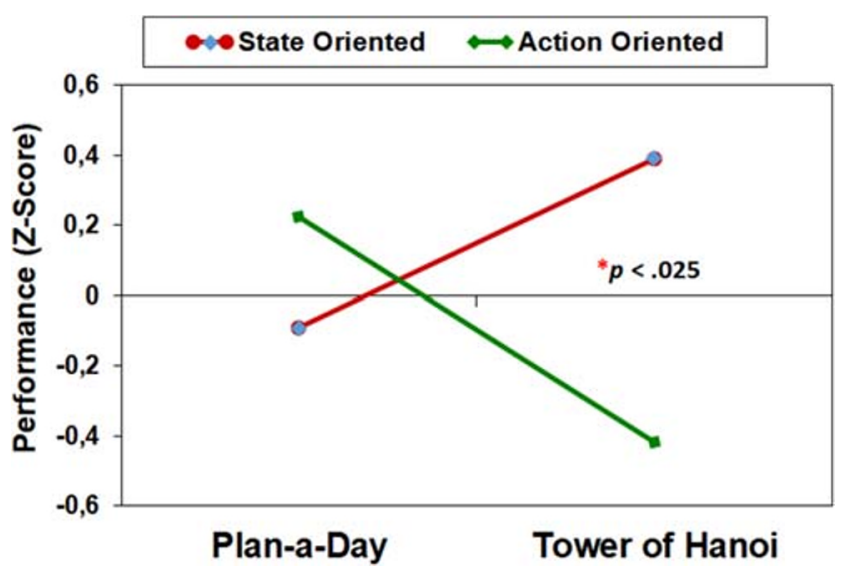

Fig. 1 Performance in the Plan-a-Day task (Percentage Correct, z-transformed) and in the Tower of Hanoi task (Number of Moves, z-transformed) as a function of individual differences in volitional efficiency. The Personality X Planning Task interaction in the ANOVA was significant $(p=.014)$. Whereas there were no significant differences between state and action oriented in Plan-a-Day $(p>.30)$, action had better performance than state oriented in the Tower of Hanoi $(p<.025)$. Better performance is indicated with positive z-scores in the Plan-a-Day task and with negative $\mathrm{z}$-scores in the Tower of Hanoi task

a warning message on a small window is displayed. There is only one correct solution for each Plan-a-Day problem. When participants notice a mistake in the execution of their plan, they can undo previous moves using the "back"-key, which is accompanied by an acoustic signal and an information window confirming the operation. Participants are instructed to avoid using the back-key by planning ahead appropriately. The main dependent variables for the Plan-a-Day test are: Number of problems solved without corrections (i.e., not using the "back"-key during the execution phase), total solution time, and number of corrections.

Tower of Hanoi We used a computer version of a five-disk version of this task. The tower of Hanoi consists of three or five rods, and a number of disks of different sizes which can slide onto any rod. The puzzle starts with the disks in a stack in ascending order of size placed on the left rod, the smallest at the top, thus making a conical shape. The goal is to move the entire stack to the right-most rod, following these rules: (1) Only one disk may be moved at a time, (2) in each move one takes the upper disk from one of the rods and slides it onto another rod, on top of the other disks that may already be present on that rod, and (c) no disk may be placed on top of a smaller disk. The minimum number of moves to solve the Tower of Hanoi puzzle is calculated by the formula $2^{n}-1$, where $n=$ number of disks. For the five-disk version that we used the minimum number of moves to solve it would be $2^{5}-1=\mathbf{3 1}$.

\section{Procedure}

The cover story was that we investigated individual differences in problem solving behavior, both simulating an everyday-life planning situation and also using a standard task, the Tower of Hanoi. We asked participants to fill out a series of questionnaires. They carried out the experiment on a PC computer in individual cabins. Participants first answered the questionnaires, including the action control scale and the VCI (including the self-determination scale). They also filled out the mood inventory. Afterwards we introduced all participants to the Plan-A-Day task. They carried out two problems of different levels of difficulty (Plan 4 and 5) for about $30 \mathrm{~min}$. There was no feedback about their performance. Subsequently all participants carried out a computer version of the 5-disk Tower of Hanoi task. This task took about 3-4 min. to be carried out, depending on the participant. After this, they filled out the mood inventory again. Finally, we thanked the participants and debriefed about the purpose of the study. The session took about $60-70 \mathrm{~min}$. Because of the fixed order of presentation, the first effortful task was Plan-a-Day whereas the task used to evaluate possible performance decrements due to ego depletion was the Tower of Hanoi.

\section{Results}

\section{Mixed-Design Analyses of the Two Tasks}

The first analysis that we computed was a Personality (state oriented, action oriented) X Planning Task (Plan-a-Day, Tower of Hanoi) mixed analysis of variance (ANOVA), with the last as within-participant factor. ${ }^{1}$ Because the main performance variable in each task was different (Plan-a-Day: "Percentage of tasks solved"; Tower of Hanoi "Number of Moves") we z-transformed the data, a method that allows a direct comparison of variables having a different metric. Results of the ANOVA indicated that Personality: $F(1$, 31 ) $=1.00, p>.30$ (n.s.), $\eta_{\mathrm{p}}^{2}=.03$, and Planning Task: $F(1$, $31)=0.14, p>.20$ (n.s.), $\eta_{\mathrm{p}}^{2}=.004$ were not significant. The predicted Personality $\mathrm{X}$ Task interaction, however, was significant: $F(1,31)=6.86, p=.014, \eta_{\mathrm{p}}^{2}=.18$ (this indicates a large effect size ${ }^{2}$ ). This interaction is depicted in Fig. 1.

A second relevant variable in the study was time spent in carrying out the tasks. Less time indicates better performance. We therefore computed a similar Personality (state oriented, action oriented) X Planning Task (Plan-a-Day, Tower of Hanoi) mixed ANOVA on time spent in the tasks. In both tasks the dependent variable was measured in seconds. Personality was

\footnotetext{
${ }^{1}$ There was a computer problem and the data from one participant (action oriented) in the Tower of Hanoi task were lost. The Tower of Hanoi results are therefore presented for 16 action oriented and 17 state oriented participants $(N=33)$.

${ }^{2}$ We used partial eta-squared $\left(\eta_{p}{ }^{2}\right)$, as indication of effect strength. It is defined as the proportion of total variation attributable to the factor partialling out (excluding) other factors from the total nonerror variance. Small, medium, and large effect sizes for an $F$-statistic have partial Eta-squared values of .01, .059 , and .138, respectively (Cohen 1992; Kirk 1996).
} 
significant: $F(1,31)=5.10, p<.05, \eta_{\mathrm{p}}^{2}=.14$. State oriented were slower $(M=145.8 \mathrm{~s}, S D=52.1)$ than action oriented participants $(M=104.8 \mathrm{~s}, S D=52.1)$. Planning Task was significant: $F(1,31)=63.14, p<.001, \eta_{\mathrm{p}}^{2}=.67$. Time spent on Plana-Day was shorter $(M=54.8 \mathrm{~s}, S D=27.6)$ than on Tower of Hanoi $(M=195.8 \mathrm{~s}, S D=99.3)$. The Personality X Task interaction tended towards significance: $F(1,31)=3.26, p=.08$, $\eta_{\mathrm{p}}^{2}=.10$ (this indicates a medium to large effect size, see Footnote 2). Further analyses of this interaction trend showed no significant difference between state and action oriented participants in time spent on the Plan-a-Day task (59.2 vs. $50.3 \mathrm{~s}$, respectively, $p>.30$ ) but showed a significant difference between state and action oriented participants in solving the Tower of Hanoi task (232.3 vs. $195.3 \mathrm{~s}$, respectively, $p<.05$ ).

\section{Plan-a-Day Task}

The ANOVAs presented in the previous section showed no differences in performance between action and state oriented participants in the Plan-a-Day task, as predicted. We further confirmed this by carrying out independent $t$-tests using the original (untransformed) measures. There were no significant differences between the groups. Percentage of tasks solved: $t(32)=0.52$, n.s., $d=0.18$ and Total solution time: $t(32)=$ 0.84 , n.s., $d=.30$ Additionally, we compared another index of performance: Number of corrections: $t(32)=1.05$, n.s., $d=.37$. Results also showed no differences between state and action oriented participants. Descriptive statistics can be seen in Table 1.

\section{Tower of Hanoi Task}

Because we used a z-transformed measure of the main dependent variable on the Tower of Hanoi task to be able to carry out the ANOVA, we carried out a further analysis with the unstransformed measure. Specifically, an independent t-test on the average Number of Moves needed to solve the task by state and action oriented participants: $t(31)=2.51$, $p=.018, d=0.90$. As shown in Fig. 2, to solve the Tower of Hanoi action oriented needed less moves $(M=56.9$; $S D=$

Table 1 Mean (\& Standard Deviations) of Performance Indices in the Plan-A-Day Task: Percentage of Tasks Solved, Mean Solution Time to Change to a Different Place within the Plan, and Number of Corrections, Separately for Action and State Oriented Participants

\begin{tabular}{llll}
\hline & Action Oriented & State Oriented & \multicolumn{1}{l}{-test } \\
\hline \% of Tasks Solved & $85.0 \%$ & $82.2 \%$ & 0.52 (n.s.) \\
& $(S D=13.5)$ & $(S D=24.9)$ & \\
Mean Solution & $51.4 \mathrm{~s}$ & $59.2 \mathrm{~s}$ & -0.84 (n.s.) \\
$\quad$ Time & $(S D=25.2)$ & $(S D=29.4)$ & \\
Number of & $19.4(S D=14.2)$ & $26.5(S D=23.6)$ & -1.05 (n.s.) \\
$\quad$ Corrections & & & \\
\hline
\end{tabular}

Tower of Hanoi: Number of Moves

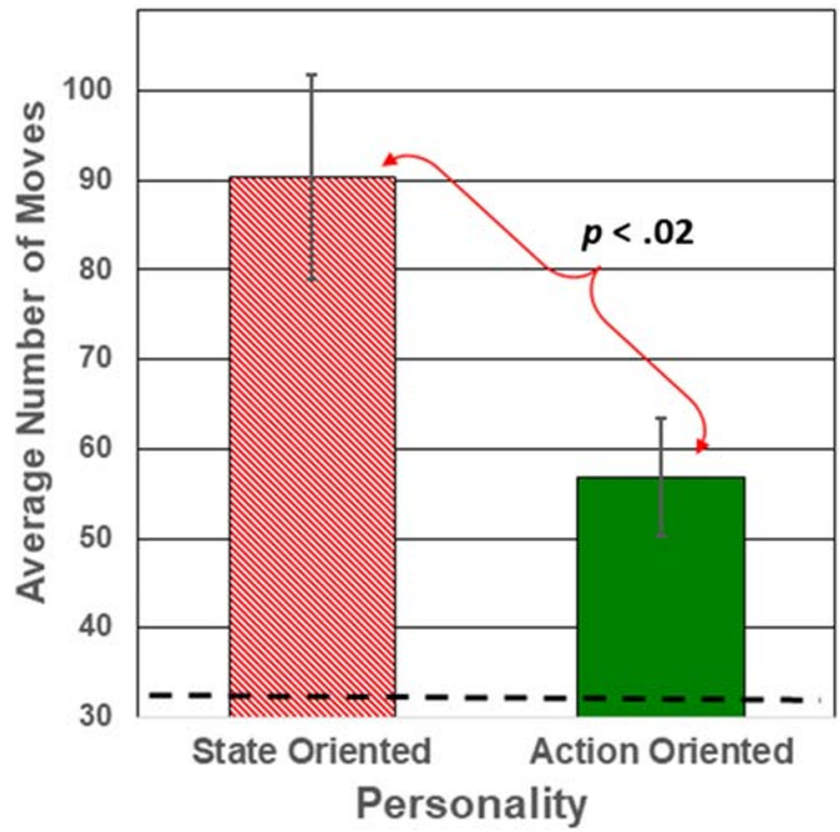

Fig. 2 Performance differences in the Tower of Hanoi task as a function of individual differences in volitional efficiency. Action oriented needed significantly less moves to solve the task than state oriented did $(p<.02)$. The dashed line indicates perfect performance ( 31 moves). The vertical lines indicate a standard error of the mean

26.5) than state oriented did $(M=90.4 ; S D=46.9)$. We also calculated an independent t-test on the Average Time taken to

Tower of Hanoi: Solution Time

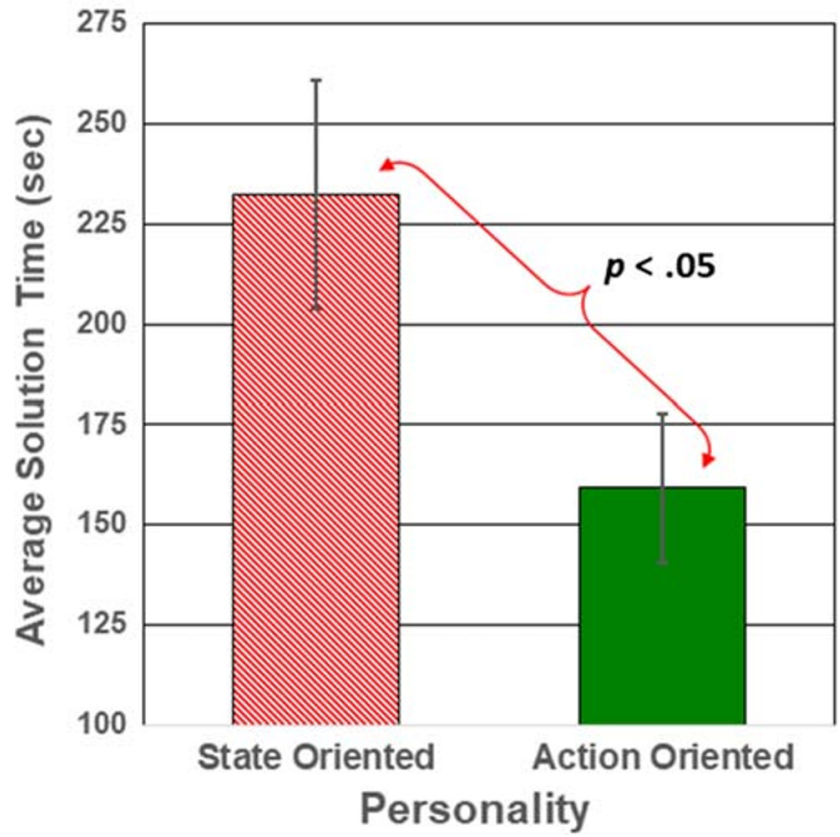

Fig. 3 Performance differences in the Tower of Hanoi task as a function of individual differences in volitional efficiency. Action oriented participants solved the task significantly faster than state oriented did $(p<.05)$. The vertical lines indicate a standard error of the mean 
solve the task (in sec) by state and action oriented participants: $t(31)=2.11, p=.043, d=0.76$. As shown in Fig. 3, to solve the Tower of Hanoi action oriented took less time $(M=159 \mathrm{~s}$; $S D=74 \mathrm{~s})$ than state oriented $\operatorname{did}(M=232 \mathrm{~s} ; S D=118 \mathrm{~s})$.

Action Orientation and Self-Determination We examined whether the self-determination measure, as a median split, would predict number of moves or solution time in the tower of Hanoi task using independent $t$ tests. Results were nonsignificant (all $t \mathrm{~s}<1$ ).

Moreover, using regression analysis, action orientation (as a continuous variable) also predicted performance in the Tower of Hanoi task. Number of moves: Beta $=-.41, t=-2.53$, $p=.017$. Solution time: $-.35, t=-2.07, p<.05$. Because higher scores in the action control scale are associated with action orientation the negative sign of the Beta coefficients indicates that action oriented required less number of moves and less time to solve the task than state oriented. The correlation between action orientation and self-determination in the experiment was positive but nonsignificant: $r(32)=+.28, p=.11$. Nonetheless, to examine whether the effects of action orientation on performance in the Tower of Hanoi task were due to a confounding with self-determination, we carried out further regression analyses entering self-determination and action orientation as simultaneous predictors in the regression. The results showed that action orientation remained a significant predictor for number of moves: Beta $=-.40, t=-2.30, p=.029$ and showed a tendency towards significance for solution time: Beta $=-.30, t=-1.69, p<.10$, after controlling statistically for the effect of self-determination. On the other hand, selfdetermination in the regression analyses was not significant ( $t \mathrm{~s}<1$; Betas $-.04 \&-.15$, for number of moves and solution time in the task, respectively). That is, the effect of action orientation on performance in the second task (Tower of Hanoi) was not due to a possible confounding with self-determination.

Affect and Performance We correlated participants' ratings of listlessness (i.e., apathy) during the experiment (i.e., averaging first and second measure) with performance on the Tower of Hanoi task (cf. Kazén et al. 2008, Experiment 1). As it may be expected, there was a positive correlation between listlessness and number of moves, $r(31)=+.40, p<.025$, and between listlessness and solution time, $r(31)=+.42, p<.025$, indicating that higher levels of listlessness were associated with worse performance in the task (more moves and longer time to solve it). On the other hand, we found a negative correlation between activation (i.e, invigoration) during the experiment and number of moves, $r(31)=-.38, p<.025$, and between activation and solution time, $r(31)=-.42, p<.025$, indicating that higher levels of activation were associated with better performance in the task (less moves and shorter time to solve it). The correlations between happiness and number of moves, $r(31)=-.09$, and of happiness and solution time, $r(31)=-.20$, were not significant. These results were obtained including all participants of the experiment.

\section{Discussion}

Action orientation is a personality dimension associated with self-regulation, whereas state orientation is associated with self-control, which is inefficient and effortful enactment of intentions especially under load (Kazén et al. 2008; Koole and Jostmann 2004; Kuhl 2000). Since the Plan-a-Day task did not entail a particular load, no significant performance differences were expected between action and state oriented participants. This expectation was confirmed: there were no signficiant differences in the percentage of tasks solved, mean solution time, or number of corrections made (see Table 1). We expected differences between action and state oriented participants in the second, Tower of Hanoi task, a conceptually similar task to the Plan-a-Day, requiring concentration, planning, and problem solving abilities. Specifically, we predicted lower performance on the Tower of Hanoi only for state oriented participants. This prediction was confirmed: In agreement with our first hypothesis, results show that action oriented participants performed significantly better than state oriented participants both in number of moves (Fig. 1) and solution times (Fig. 2) needed to solve the Tower of Hanoi. This finding replicates Jostmann and Gieselmann (2014) who also found deficits by state-oriented participants in this task, showing that not only left prefrontal brain patients have a deficit in the Tower of Hanoi (Morris et al. 1997), but they are also found in normal (state oriented) persons under demanding or ego-depletion conditions.

Our findings go beyond the results of Jostmann and Gieselmann (2014) because (a) we used a two-task paradigm and (b) had a five-disk version of the Tower of Hanoi. In addtion, we show that individual differences in performance deficits cannot be attributed to differences in autonomy: Individual differences in self-determination (as an indicator of autonomy) did not predict performance in the Tower of Hanoi task, and the results remained equally strong for action orientation when action orientation and self-determination were entered simultaneously in the regression analyses predicting performance. This suggest that although action orientation and self-determination are moderately related $(r=.28$, n.s.), they are independent and only the dimension of action vs. state orientation predicted ego-depletion or invigoration effects in this experiment.

Our second hypothesis was confirmed: Listlessness correlated with lower performance (number of moves and solution time), and activation with higher performance (number of moves and solution times) in the Tower of Hanoi. Notably, we found small negative but not significant correlations between happiness and performance in this task. This suggests 
that happiness, in contrast to activation, may not be conducive to carry out efficiently tasks requiring planning and analytic processing. According to PSI theory (Kuhl 2000), intention memory is needed whenever planning or a difficult intention has to be postponed and it is activated under conditions in which positive affect is low and not high (Goschke and Kuhl 1993; Kazén et al. 2008; Kuhl \& Kazén, 1999).

Gröpel et al. (2014) presented converging evidence that the personality dimension of action vs. state orientation moderates ego-depletion effects. They found that actionoriented participants performed better on a concentration task than state-oriented after a strenuous physical exercise (Experiment 1), had higher acuity on the critical fusion frequency test after a vigilance test (Experiment 2), and had better Stroop performance after a presumably depleting sensorimotor task (Experiment 3). In each study they found no differences between action and state oriented participants on the first task. This pattern of results is consistent with our model emphasizing individual differences in executive control mode (i.e., action vs. state orientation) as a moderator of depletion effects.

The present experiment shows that ego depletion does not always occur after exerting self-control in a resourcedemanding activity. The meta-analysis of Hagger et al. (2010) indicates that ego-depletion is a reliable phenomenon, although there are different explanations proposed to explain it (see pp. 517-519). How can one avoid ego-depletion? One possibility is through rest and sleep (Baumeister et al. 2000). Other moderators preventing ego depletion, are implementation intentions (Webb and Sheeran 2004), task motivation (Muraven and Slessareva 2003), and training (Muraven, Baumeister, \& Tice, 1999; see Hagger et al. 2010, pp. 501502). As indicated in the introduction, an additional moderator is the sense of autonomy, or self-determination, that is, having participants choose the to-be-carried out tasks instead of imposing them externally. Only in the latter case are egodepletion effects to be expected (Moller et al. 2006; Muraven 2008; Ryan and Deci 2008)

The construct of Action versus State Orientation from Kuhl seems to be similar to the Locus of Control construct postulated by Rotter (1966). ${ }^{3}$ That is, it may be expected that action oriented persons have an internal whereas state oriented persons have an external locus of control. Are there differences between these constructs? Kuhl (1982) noted that locus of control refers to how much control people think they have, whereas action orientation relates to the amount of control that they actually exert. For example, a person may think that she can control everything but make little use of this imagined potential because she is either not interested in using it or not able to use it. Empirical findings support this logic in that

\footnotetext{
${ }^{3}$ We thank an anonymous reviewer for making us aware of a possible relation between action vs. state orientation and locus of control.
}

the correlation between Rotter's scale and the action control scale has been found to be very low (e.g., $r=.04$; Kuhl 1982).

\section{Limitations}

First, participants did not have any choice in selecting the tasks to be performed, which restricted their self-determination. Perhaps allowing participants to choose a particular version of the tasks to be performed would help overcoming possible ego-depletion effects shown by state oriented persons. Second, the number of participants should be increased in future experiments. Although the $p$-values of the $t$-tests comparing state and action oriented participants on each of the dependent variables of the Plan-a-Day task were far from being significant (see Table 1), the effect sizes of those independent $t$-tests ranged from low (percentage of tasks solved), higher than small (total solution time), to close to medium (number of corrections), according to Cohen's $d$. With a larger sample the difference between state and action oriented persons may have turned out to be significant in this task.

However, our main hypothesis focused on finding a significant interaction between Personality and Planning task, expecting no performance differences between the groups of participants in the first, Plan-a-Day task, but to find them in the second, Tower-of Hanoi task, because state but not action oriented participants are expected to deplete their resources in carrying out the first strenuous task. The results of the two mixed-design ANOVAs supported our expectations and the significant and fast significant interactions obtained showed a large $\left(\eta_{\mathrm{p}}^{2}=.18\right)$ and a medium to large effect size $\left(\eta_{\mathrm{p}}^{2}=.10\right)$, respectively (see Footnote 2 ). Note that this was not a "no effect"-hypothesis, because our focus was on the interaction between Personality and Planning Task, and not only on not finding differences between the groups of participants in the first task. There is no a-priori reason to expect that state and action oriented participants would differ in their ability to perform well in the Plan-a-Day or in the Tower of Hanoi tasks. It is well-know that they do not differ in intelligence, but they differ in volitional abilities (Diefendorff et al. 2000; Kuhl and Beckmann 1994).

Third, all of our participants were healthy undergraduate students. Low performance in the Tower of Hanoi has been linked to deficits in executive processes, which are shown by left-prefrontal brain patients (Morris et al. 1997). One could investigate whether these patients - compared to healthy participants - are especially vulnerable to ego-depletion effects using a two-task paradigm like we used here.

\section{Conclusion}

We show in this experiment that action orientation is an important moderator of ego-depletion vs. invigoration effects, 
independent from individual differences in self-determination. State oriented participants show ego-depletion in the Tower of Hanoi task after a strenuous first "Plan-a-Day" task, whereas action oriented do not. According to PSI theory (Kuhl 2000, 2001) self-control, the preferred mode of state oriented persons under load, is an autocratic form of executive control associated with activation of intention memory and effortful enactment of intentions, suppressing all other needs of the individual. Self-regulation, the preferred mode of action oriented persons, is a democratic form of executive control, associated with extension memory, which integrates multiple needs in carrying out our intentions.

Funding Open Access funding enabled and organized by Projekt DEAL.

\section{Compliance with Ethical Standards}

All procedures performed in studies involving human participants were in accordance with the ethical standards of the institutional and/or national research committee and with the 1964 Helsinki declaration and its later amendments or comparable ethical standards.

Conflict of Interest On behalf of all authors, the corresponding author states that there is no conflict of interest.

Informed Consent was obtained from all individual participants included in the study.

Open Access This article is licensed under a Creative Commons Attribution 4.0 International License, which permits use, sharing, adaptation, distribution and reproduction in any medium or format, as long as you give appropriate credit to the original author(s) and the source, provide a link to the Creative Commons licence, and indicate if changes were made. The images or other third party material in this article are included in the article's Creative Commons licence, unless indicated otherwise in a credit line to the material. If material is not included in the article's Creative Commons licence and your intended use is not permitted by statutory regulation or exceeds the permitted use, you will need to obtain permission directly from the copyright holder. To view a copy of this licence, visit http://creativecommons.org/licenses/by/4.0/.

\section{References}

Baumeister, R. F., Bratslavsky, E., Muraven, M., \& Tice, D. M. (1998). Self-control depletion: Is the active self a limited resource? Journal of Personality and Social Psychology, 74, 1252-1265. https://doi. org/10.1037//0022-3514.74.5.1252.

Baumeister, R. F., Muraven, M., \& Tice, D. M. (2000). Ego depletion: A resource model of volition, self-regulation, and controlled processing. Social Cognition, 18, 130-150. https://doi.org/10.1521/soco. 2000.18.2.130.

Baumeister, R. F., Schmeichel, B. J., \& Vohs, K. D. (2007). Selfregulation and the executive function: The self as controlling agent. In Social psychology: Handbook of basic principles (2nd ed., pp. 516-539). New York: The Guilford Press.

Beckmann, J., \& Kuhl, J. (1984). Altering information to gain action control: Functional aspects of human information processing in decision making. Journal of Research in Personality, 18(2), 224 237. https://doi.org/10.1016/0092-6566(84)90031-X.

Cohen, J. (1992). A power primer. Psychological Bulletin, 112, 155-159. https://doi.org/10.1037/0033-2909.112.1.155.

Dang, J., Xiao, S., Shi, Y., \& Mao, L. (2015). Action orientation overcomes the ego depletion effect. Scandinavian Journal of Psychology, 56(2), 223-227. https://doi.org/10.1111/sjop.12184.

Deci, E. L., \& Ryan, R. M. (1985). The general causality orientations scale: Self-determination in personality. Journal of Research in Personality, 19(2), 109-134. https://doi.org/10.1016/00926566(85)90023-6.

Diefendorff, J. M., Hall, R. J., Lord, R. G., \& Strean, M. L. (2000). Action-state orientation: Construct validity of a revised measure and its relationship to work-related variables. Journal of Applied Psychology, 85, 250-263. https://doi.org/10.1037/0021-9010.85.2. 250.

Fuhrmann, A., \& Kuhl, J. (1998). Maintaining a healthy diet: Effects of personality and self-reward versus self-punishment on commitment to and enactment of self-chosen and assigned goals. Psychology and Health, 13, 651-686. https://doi.org/10.1080/08870449808407423.

Funke, J., \& Krüger, T. (1993). "Plan-A-Day" (PAD). Psychological Institute of the University of Bonn. Bonn: Germany.

Funke, J., \& Krüger, T. (1995). "Plan-A-day": Konzeption eines modifizierbaren instruments zur Führungskräfte-Auswahl sowie erste empirische Befunde ["plan-A-day": Design of a modifiable instrument for selecting managerial personnel and first empirical results]. In J. Funke, T. Krüger, \& A. Fritz (Eds.), Neue Konzepte und Instrumente zur Planungsdiagnostik (pp. 97-120). Bonn: Deutscher Psychologen Verlag.

Goschke, T., \& Kuhl, J. (1993). Representation of intentions: Persisting activation in memory. Journal of Experimental Psychology: Learning, Memory, and Cognition, 19(5), 1211-1226. https://doi. org/10.1037/0278-7393.19.5.1211.

Gröpel, P., Baumeister, R. F., \& Beckmann, J. (2014). Action versus state orientation and self-control performance after depletion. Personality and Social Psychology Bulletin, 40(4), 476-487. https://doi.org/10. $1177 / 0146167213516636$.

Heckhausen, H., \& Strang, H. (1988). Efficiency under record performance demands: Exertion control - an individual difference variable? Journal of Personality and Social Psychology, 55(3), 489498. https://doi.org/10.1037/0022-3514.55.3.489

Hagger, M. S., Wood, C., Stiff, C., \& Chatzisarantis, N. L. D. (2010). Ego depletion and the strength model of self-control: A meta-analysis. Psychological Bulletin, 136, 495-525. https://doi.org/10.1037/ a0019486.

Holt, D. V., Rodewald, K., Rentrop, M., Funke, J., Weisbrod, M., \& Kaiser, S. (2011). The plan-a-day approach to measuring planning ability in patients with schizophrenia. Journal of the International Neuropsychological Society, 17(02), 327-335. https://doi.org/10. 1017/S1355617710001712.

Jostmann, N. B., \& Gieselmann, A. (2014). When you have to climb downhill to reach the top. Experimental Psychology, 61(5), 394401. https://doi.org/10.1027/1618-3169/a000260.

Jostmann, N. B., \& Koole, S. L. (2007). On the regulation of cognitive control: Action orientation moderates the impact of high demands in Stroop interference tasks. Journal of Experimental Psychology. General, 136(4), 593-609. https://doi.org/10.1037/0096-3445.136. 4.593.

Kazén, M., Kaschel, R., \& Kuhl, J. (2008). Individual differences in intention initiation under demanding conditions: Interactive effects of state vs. action orientation and enactment difficulty. Journal of Research in Personality, 42, 693-715. https://doi.org/10.1016/j.jrp. 2007.09.005.

Kazén, M., Kuhl, J., \& Leicht, E.-M. (2015). When the going gets tough...: Self-motivation is associated with invigoration and fun. 
Psychological Research, 79(6), 1064-1076. https://doi.org/10. 1007/s00426-014-0631-z.

Kehr, H. M. (2004). Emplicit/explicit motive discrepancies and volitional depletion among managers. Personality and Social Psychology Bulletin, 30(3), 315-327. https://doi.org/10.1177/ 0146167203256967.

Kirk, R. E. (1996). Practical significance: A concept whose time has come. Educational and Psychological Measurement, 56, 746-759. https://doi.org/10.1177/0013164496056005002.

Koole, S. L., \& Jostmann, N. B. (2004). Getting a grip on your feelings: Effects of action orientation and external demands on intuitive affect regulation. Journal of Personality and Social Psychology, 87(6), 974-990. https://doi.org/10.1037/0022-3514.87.6.974.

Koole, S. L., Jostmann, N. B., \& Baumann, N. (2012). Do demanding conditions help or hurt self-regulation? Social and Personality Psychology Compass, 6(4), 328-346. https://doi.org/10.1111/j. 1751-9004.2012.00425.x.

Kuhl, J. (1982). Action vs. state orientation as a mediator between motivation and action. In W. Hacker, W. Volpert, \& M. von Cranach (Eds.), Cognitive and motivational aspects of action (pp. 67-85). Berlin, GDR: VEB Deutscher Verlag der Wissenschaften.

Kuhl, J. (1994). Action and state orientation: Psychometric properties of the action control scales (ACS-90). In J. Kuhl \& J. Beckmann (Eds.), Volition and personality: Action versus state orientation (pp. 4759). Göttingen: Hogrefe.

Kuhl, J., \& Kazén, M. (1999). Volitional facilitation of difficult intentions: Joint activation of intention memory and positive affect removes Stroop interference. Journal of Experimental Psychology: General, 128, 382-399. https://doi.org/10.1037/0096-3445.128.3. 382

Kuhl, J. (2000). The volitional basis of personality systems interaction theory: Applications in learning and treatment contexts. International Journal of Educational Research, 33(7-8), 665-703. https://doi.org/10.1016/S0883-0355(00)00045-8.

Kuhl, J. (2001). Motivation und Persönlichkeit: Interaktionen psychischer Systeme [motivation and personality: Interactions of mental systems]. Göttingen, Germany: Hogrefe.

Kuhl, J., \& Beckmann, J. (1994). Volition and personality: Action versus state orientation. Göttingen, Germany: Hogrefe.

Kuhl, J., \& Fuhrmann, A. (1998). Decomposing self-regulation and selfcontrol: The volitional components checklist. In J. Heckhausen \& C. Dweck (Eds.), Life span perspectives on motivation and control (pp. 15-49). Mahwah: Erlbaum.

Moller, A. C., Deci, E. L., \& Ryan, R. M. (2006). Choice and ego-depletion: The moderating role of autonomy. Personality and Social Psychology Bulletin, 32, 1024-1036. https://doi.org/10.1177/ 0146167206288008.

Morris, R. G., Miotto, E. C., Feigenbaum, J. D., Bullock, P., \& Polkey, C. E. (1997). The effect of goal-subgoal conflict on planning ability after frontal- and temporal-lobe lesions in humans. Neuropsychologia, 35(8), 1147-1157. https://doi.org/10.1016/ S0028-3932(97)00009-2.
Muraven, M., Baumeister, R. F., \& Tice, D. M. (1999). Longitudinal improvement of self-regulation through practice: Building selfcontrol strength through repeated exercise. The Journal of Social Psychology, 139(4), 446-457. https://doi.org/10.1080/ 00224549909598404

Muraven, M. (2008). Autonomous self-control is less depleting. Journal of Research in Personality, 42(3), 763-770. https://doi.org/10.1016/ j.jrp.2007.08.002.

Muraven, M., \& Slessareva, E. (2003). Mechanisms of self-control failure: Motivation and limited resources. Personality and Social Psychology Bulletin, 29, 894-906. https://doi.org/10.1177/ 0146167203029007008.

Muraven, M., Gagné, M., \& Rosman, H. (2008). Helpful self-control: Autonomy support, vitality, and depletion. Journal of Experimental Social Psychology, 44(3), 573-585. https://doi.org/ 10.1016/j.jesp.2007.10.008.

Rotter, J. (1966). Generalized expectancies for internal versus external control of reinforcement. Psychological Monographs: General and Applied, 80(1), 1-28. https://doi.org/10.1037/h0092976.

Ryan, R. M., \& Connell, J. P. (1989). Perceived locus of causality and internalization: Examining reasons for acting in two domains. Journal of Personality and Social Psychology, 57(5), 749-761. https://doi.org/10.1037/0022-3514.57.5.749.

Ryan, R. M., \& Deci, E. L. (2000). Self-determination theory and the facilitation of intrinsic motivation, social development, and wellbeing. American Psychologist, 55, 68-78. https://doi.org/10.1037/ 0003-066X.55.1.68.

Ryan, R., \& Deci, E. (2008). From ego depletion to vitality: Theory and findings concerning the facilitation of energy available to the self. Social and Personality Psychology Compass, 2, 702-717. https:// doi.org/10.1111/j.1751-9004.2008.00098.x.

Tice, D. M., \& Bratslavsky, E. (2000). Giving in to feel good: The place of emotion regulation in the context of general self-control. Psychological Inquiry, 11(3), 149-159. https://doi.org/10.1207/ S15327965PLI1103 03.

Vonasch, A., Vohs, K., Ghosh, A. P., \& Baumeister, R. (2017). Ego depletion induces mental passivity: Behavioral effects beyond impulse control. Motivation Science, 3(4), 321-336. https://doi.org/10. 1037/mot0000058.

Watson, D., Clark, L. A., \& Tellegen, A. (1988). Development and validation of brief measures of positive and negative affect: The PANAS scales. Journal of Personality and Social Psychology, 54, 1063-1070.

Webb, T. L., \& Sheeran, P. (2004). Identifying good opportunities to act: Implementation intentions and cue discrimination. European Journal of Social Psychology, 34, 407-419. https://doi.org/10. 1002/ejsp.205.

Publisher's Note Springer Nature remains neutral with regard to jurisdictional claims in published maps and institutional affiliations. 